\title{
Design of Intelligent Stadiums Management System Based on ASP.NET
}

\author{
Jing NING \\ Physical Research Department, Hebei University, Baoding, Hebei, China, 071002 \\ 16736369@qq.com
}

\begin{abstract}
This paper analyzes the application requirements of stadium, and $B / S$ architecture is used to design of stadium with intelligent management system, including five modules: systematic management, site management, membership management, sparring management and device management. SQLServer2005 database management system is used to establish a database system so that we can achieve seamless and efficient combination of ASP.NET applications. Finally, the basic module functions of the system were tested and the result shows that the system functions achieve the desired goals. It performed well and could improve efficiency greatly.
\end{abstract}

Keywords: Stadiums; ManagementSystem; ASP.NET

\section{Introduction}

The stadiums play a very important role in organizing large-scale game and it will be greatly significant in the development of sports, but because of many objective reasons, the current domestic sports venues intelligent level is not high, at the same time, Chinese large numbers of gyms have a lot of deficiencies in the informational technology. Recently, the number of stadium not only has increased, but the scale also expanded, which led the stadium management became seriously difficult.Consequently, it is high time to establish web-based database technology gymnasium intelligent management systems. With the help of intelligent management system, we can greatly improve the efficiency of the staff, and achieve stadium information management. Inaddition, it also enables consumers to enjoy the convenience brought by information technology.

Since the beginning of the 1980s, many countries in the design of the stadium returned gradually from the blind pursuit of large-scale shift to advanced equipment, versatile, comfortable and safe, and the direction of modern eco-friendly development. In 1984, in the twenty-third Olympic Games was held by Los Angeles, it's the first time to combined intelligent system with sport, the information systems that based on communications and computer played the role of information and services in the Olympic Games. It is the first attempt and is successful, it achieved the world recognition, creating Olympic history as well as the use of information and communication technology, which the IOC gave provision that the level of information and communication systems and services for the Olympic Games must have a set of technical services index ${ }^{[1]}$.

Our stadium intelligent system is inspired by and derived from intelligent building, and it is our country unique. Construction of sports at all levels of the information system is the driving force and the foundation of our gymnasium intelligent system development. At the Sixth National Games held in Guangzhou in 1987, the first real use of computer technology to deal with the results and publish information. Since then, information systems has become a national comprehensive sports held in the requirements ${ }^{[2]}$. In 1997, Eighth National Games stadium brought vigorous application of intelligent system, for which the new Shanghai Stadium is the first stadium with a variety of intelligent system by China established. It will combine with sports demand for information to achieve a 
stadium of intelligence and information, which has opened up the way of construction of a modern stadium ${ }^{[3]}$. At the end of twentieth century, Wuhan stadium construction referred to the former experience, increasing video surveillance systems and other additional ticket sale system to enrich the content of the intelligent system with innovative ideas, so that the construction of a modern stadium appeared a new situation ${ }^{[4]}$. Nanjing Olympic Sports Center and other construction for the service of 2008 Beijing Olympic Games Stadium on large-scale use of modern technology has greatly promoted the stadium toward more intelligent systems deepen and refine the direction of development. At this time, the intelligent building of the stadium reached an unprecedented climax.

\section{System Requirements Analysis}

\subsection{Businesses Process Analysis}

There are different kinds of objects of participation in the stadium, including customers, use of the gym staff, management workers, venues, service workers ${ }^{[5]}$. All the properties of the client come to customer information summary table, which mainly contains the customer's name, address, age and so on. After having a certain understanding of the table, it can help to communicate with customers and work. In the stadium, since the staff is more complex, it will result in a relatively large amount of information, and it is very important to take the relevant information intensive announced.

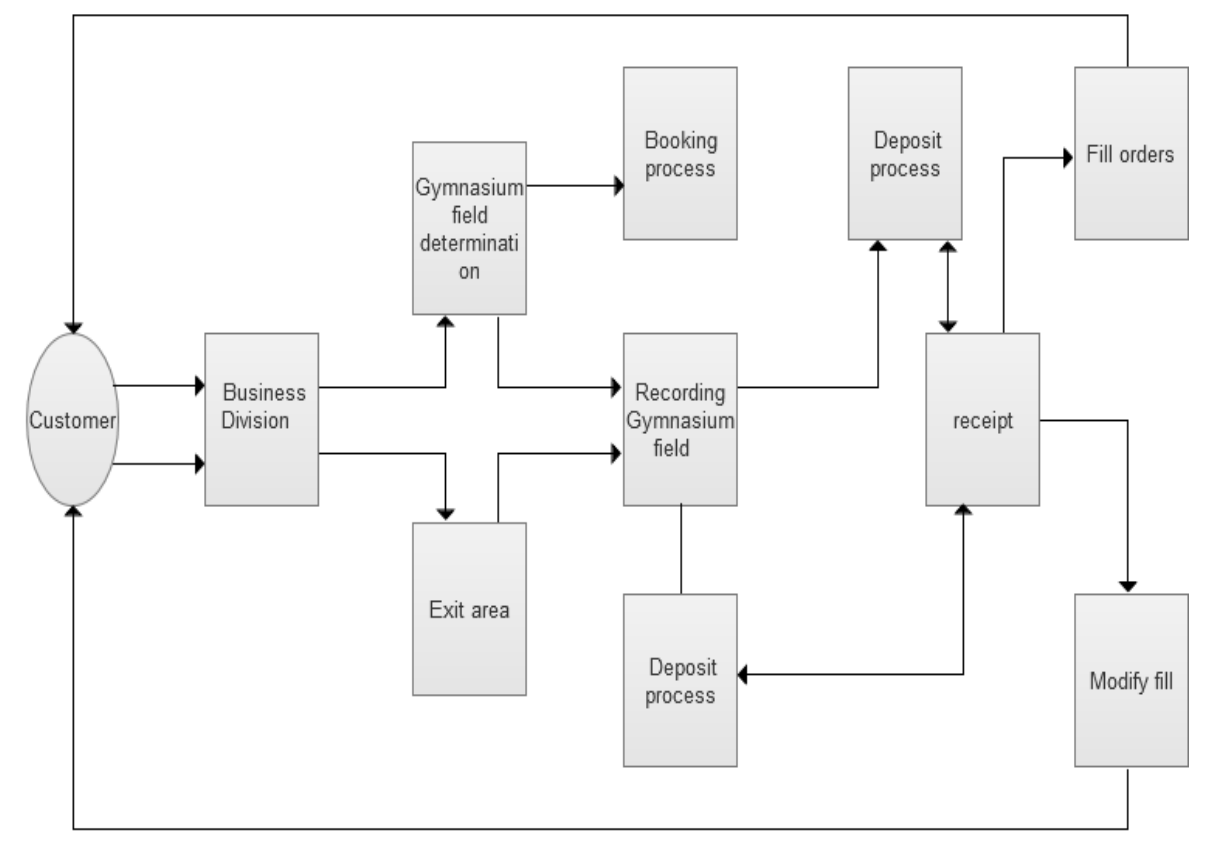

Figure 1. Stadium Management Business Flow Char

As shown in Figure1, only one manager can manage the completion of the site and funds. Management staff in view of the business after the requisition is processed, and then according to the query field records to determine the effectiveness of a single application, if it's not, you need to return the application to the customer.

\subsection{System Data Flow Analysis}

The use of data flow diagrams stadiums linked different business processes each other, through calculating the complete processing of information, which can indicate the direction of information flow in a system, as well as storage and so on. Top-level data flow diagram shown in Figure 2. 


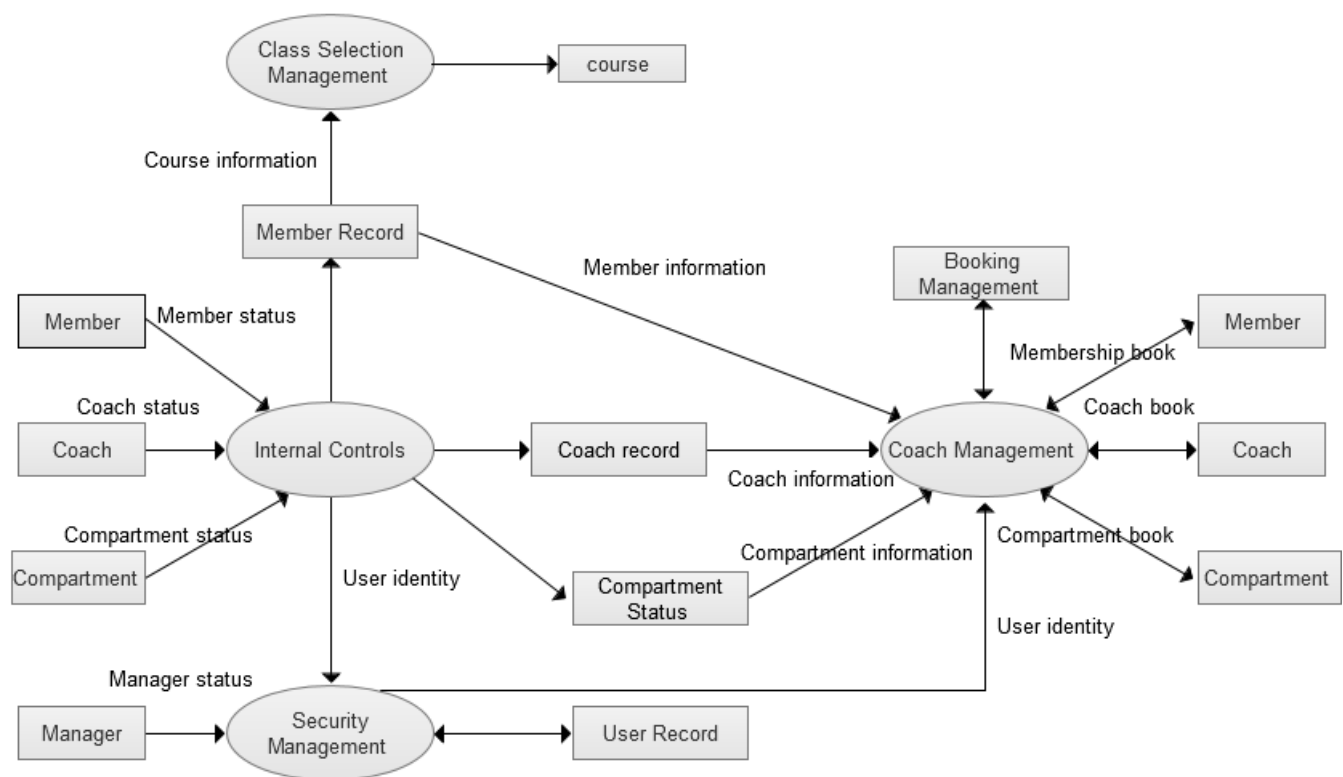

Figure 2.Top-level Data Flow Diagram

"Internal management" to refine the data flow diagram shown in Figure 3.
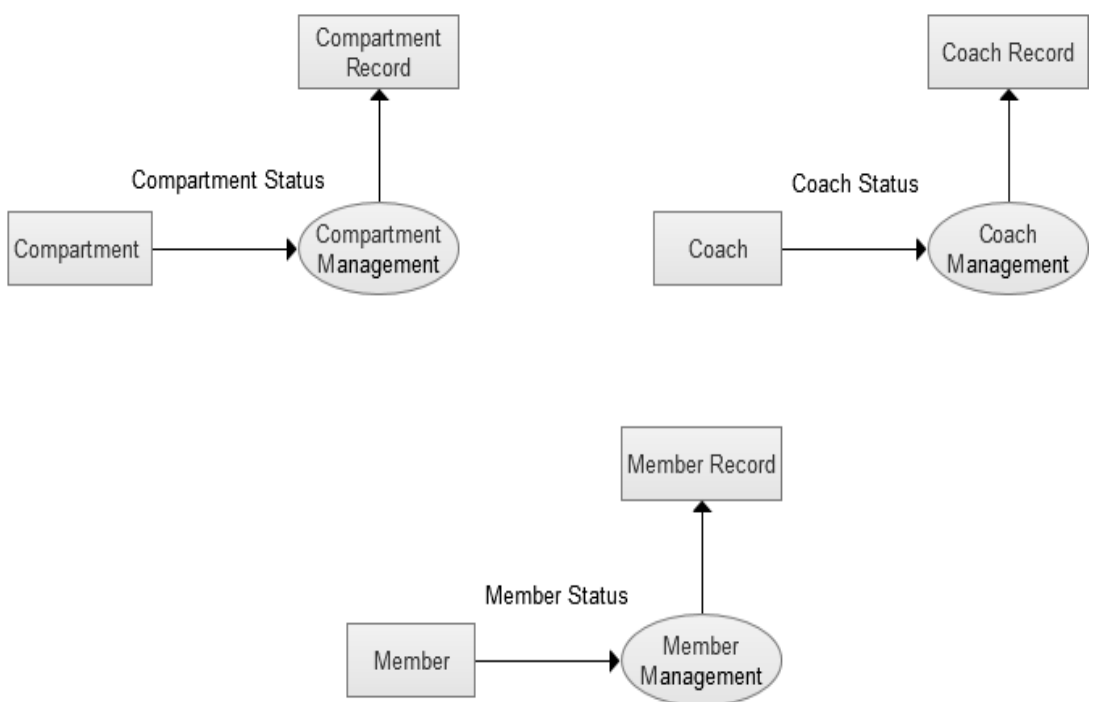

Figure 3. "Internal Management" to Refine Data Flow Diagram

There are a lot of areas in the process of stadium site management business following: treatment, processing and handling of financial business and other venues, and the main information databases, library card database which related to the billing information, to deal with the orders and receipt processing is the last $\operatorname{step}^{[6]}$. As shown in Figure 4: 


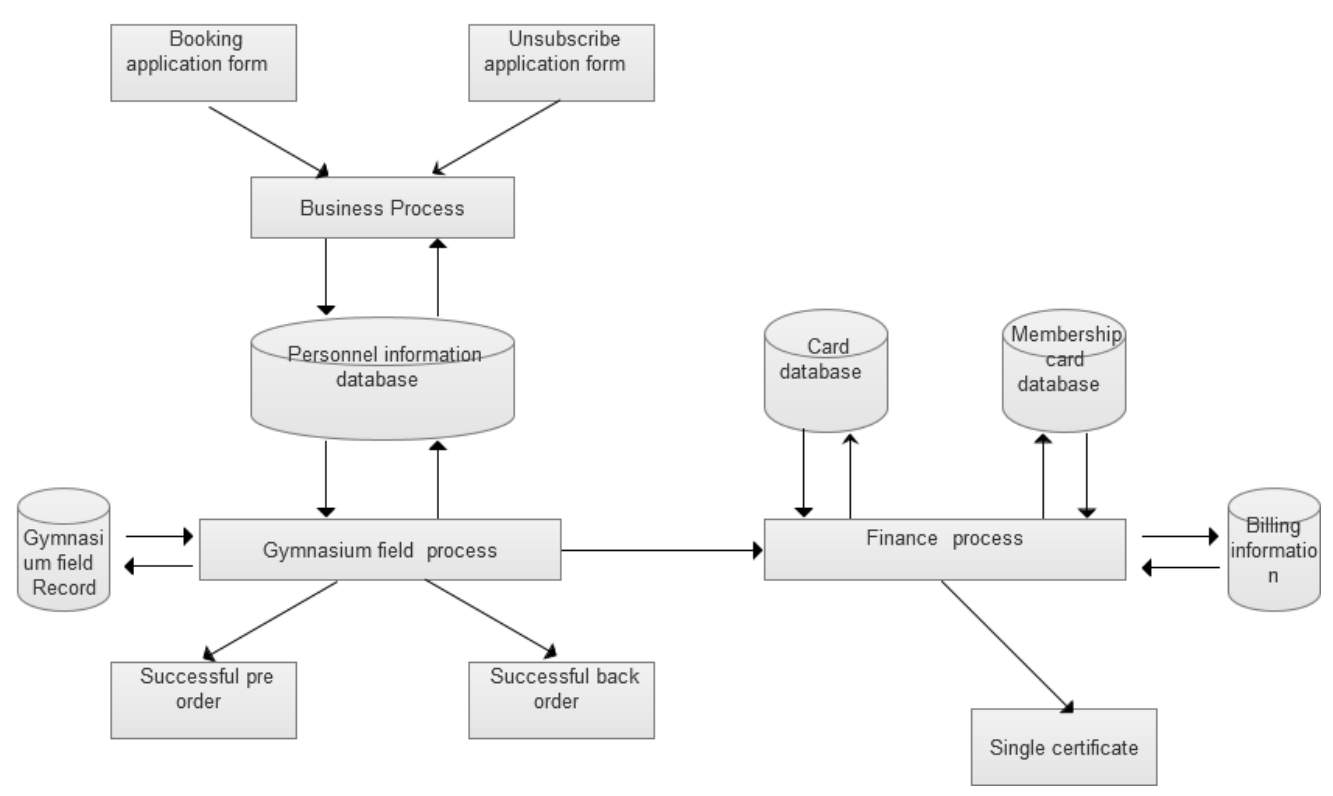

Figure 4. Site Management Data Flow Diagram

\section{The Overall System Design of Framework}

\subsection{System Architecture Design}

The design of the structure stadium management information system through B / S which is a structure by the browser, Web server and database server consisting of 3-layer model as shown in Figure 5:

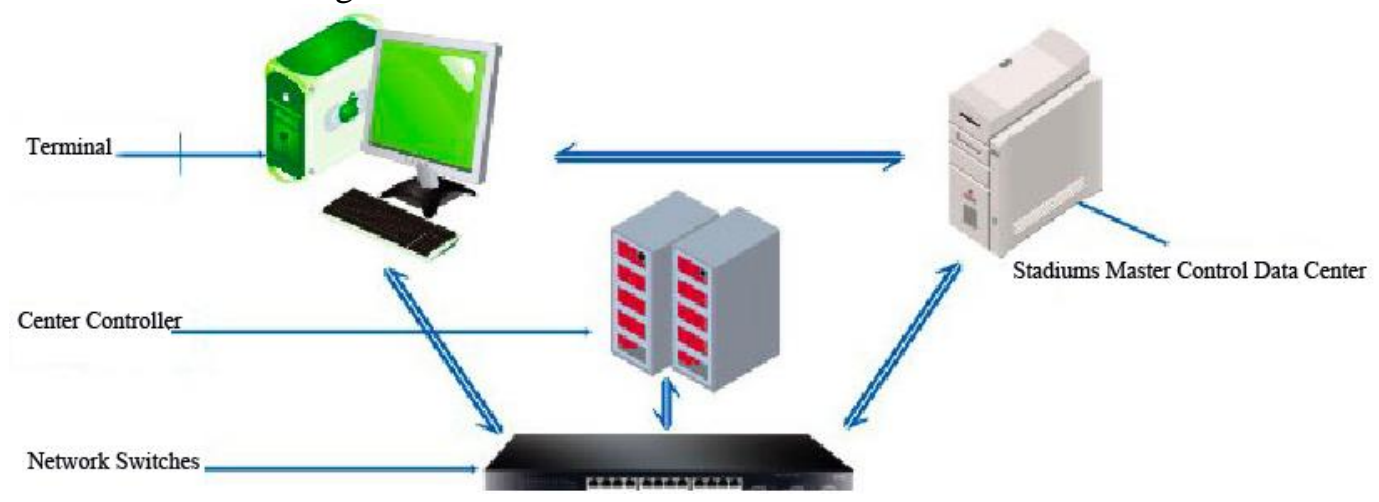

Figure 5. System Topology

\subsubsection{B / S Architecture}

The system used B / S system structure, which is mainly through the browser, Web server and the data server three levels of composition, structure diagram shown in Figure 6 , it is based on TCP / IP protocol, the most important job is to complete server-side applications maintenance and development. Therefore, the data are basically exist on the data server, the browser can submit the completed form to work only through the form of Form POST and GET data to complete the operation. The main work process is as follows: at the beginning with which we need to complete forms to fill in, when submitting HTTP, browser analysis forms, as long as the content of the request for an access server after calling ADO.NET complete the next operation, the final result can be displayed by the help of browser back to the server. 

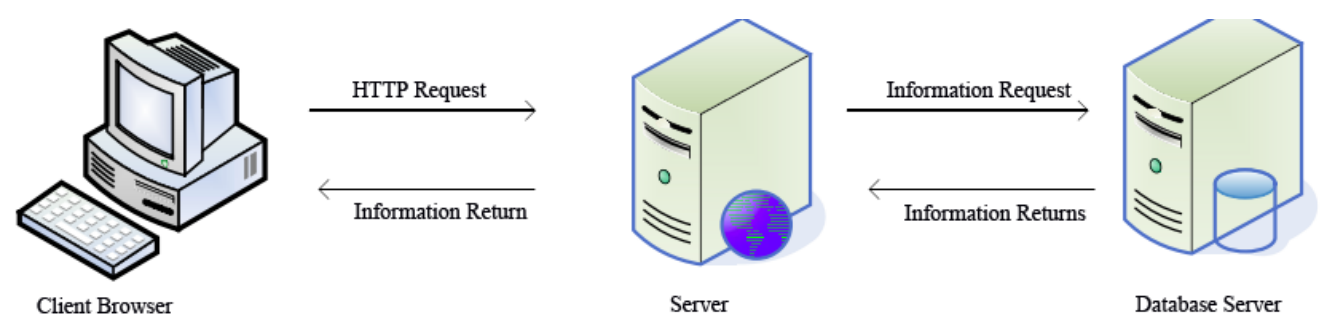

Figure 6. Based on the Three-tier Structure of B / S Mode

After the server using MS SQL Server 2005 database server software, at the time of processing data in terms of flexibility or security, etc., which all has greatly improved.

\subsubsection{ASP.NET Technology}

ASP.NET is part of the .NET Framework, as well as the revised versions of ASP, which solves many of the problems of ASP. C \# can be used to create ASP.NET pages. ASP.NET pages are structured. Each page is a .NET class inherits System.Web.UI.Page classes can override methods in a series of lifetime Page object calls. It can function in the clear meaning of the event handler as a page, thus, ASP.NET is easier to understand. It is obviously to see ASP.NET improvement on performance.

ASP NET is a three-tier structure of natural systems: UI layer, business logic layers and data layers, which structure shown in Figure 7:

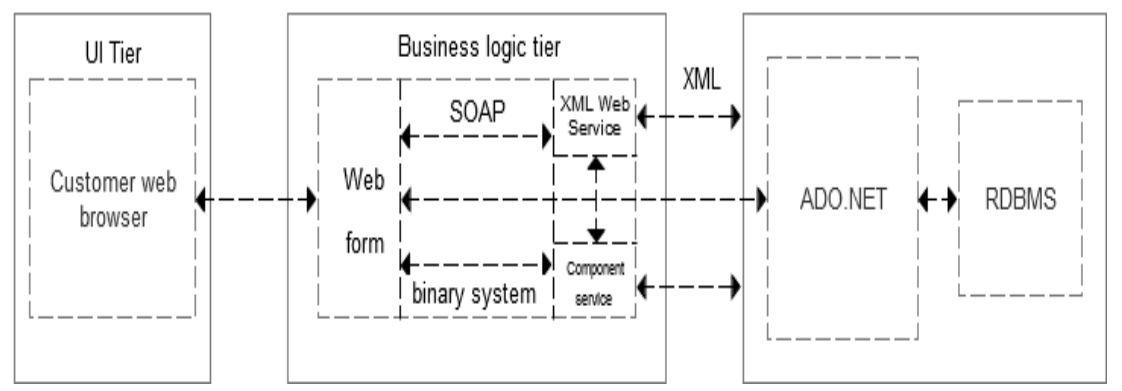

Figure 7 .ASP. NET System Architecture

Where in the UI layer is responsible for interacting with the user, receiving input from user data and presented to the customer from the server. Business logic layer is responsible for receiving requests coming from the browser and convey the request to the data layer, while send the request processing results to the browser. It consists of Web forms, XML Web Services and Component Services components. Web forms not at the heart of the foundation of ASP. NET, as well as present data and information to customers, but also respond to and deal with customers and display interactive Web forms generated information and data base.

Business logic layer is responsible for all business logic processing; in this paper it is responsible for receiving UI layer pass over the parameters. According to the parameters, to determine their own business rules, convenient and practical for programming in logic layer adds a more low-level components sqldb, responsible for database-related storage operations through the following code:

Publicclasssqldb

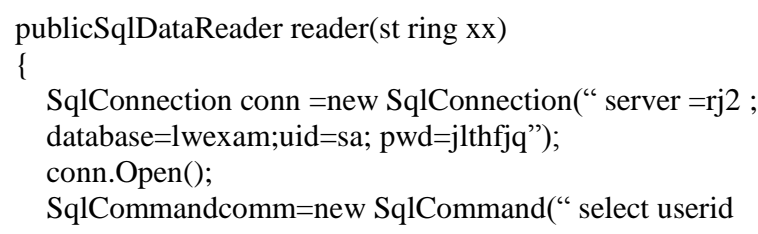




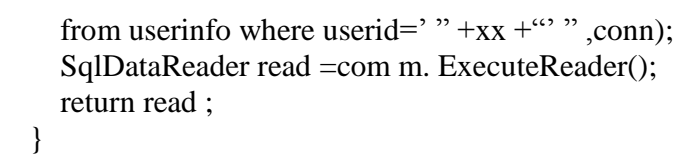

Data layer is formed by ADO. NET manipulate the data to provide data services to business logic layer, such as storing data operation results, returning search results and other data. In the data layer, in order to improve application performance, stored procedures can be introduced.

\subsubsection{ADO.NET Components}

ADO.NET is an integral part of the .NET Framework,as well as a data-access technology that allows applications to connect to the data store, and manipulate data stored in which in a variety of ways.

The "provider" of ADO.NET is some components that know how to interact with specific data and storage facilities (for example, a provider of SQL Server to interact with other providers to interact with an Oracle database). Which comprises four core objects: the first one is the Connection object that is used to establish a connection with particular data source); the second is the Command object used to execute commands on the data source; the third is a Data Reader object, which used for reading only in and read-only data stream from the data source; the fourth is the Data Adapter object that used to fill the Data Set and resolves updates with the data source. As shown in FIG.

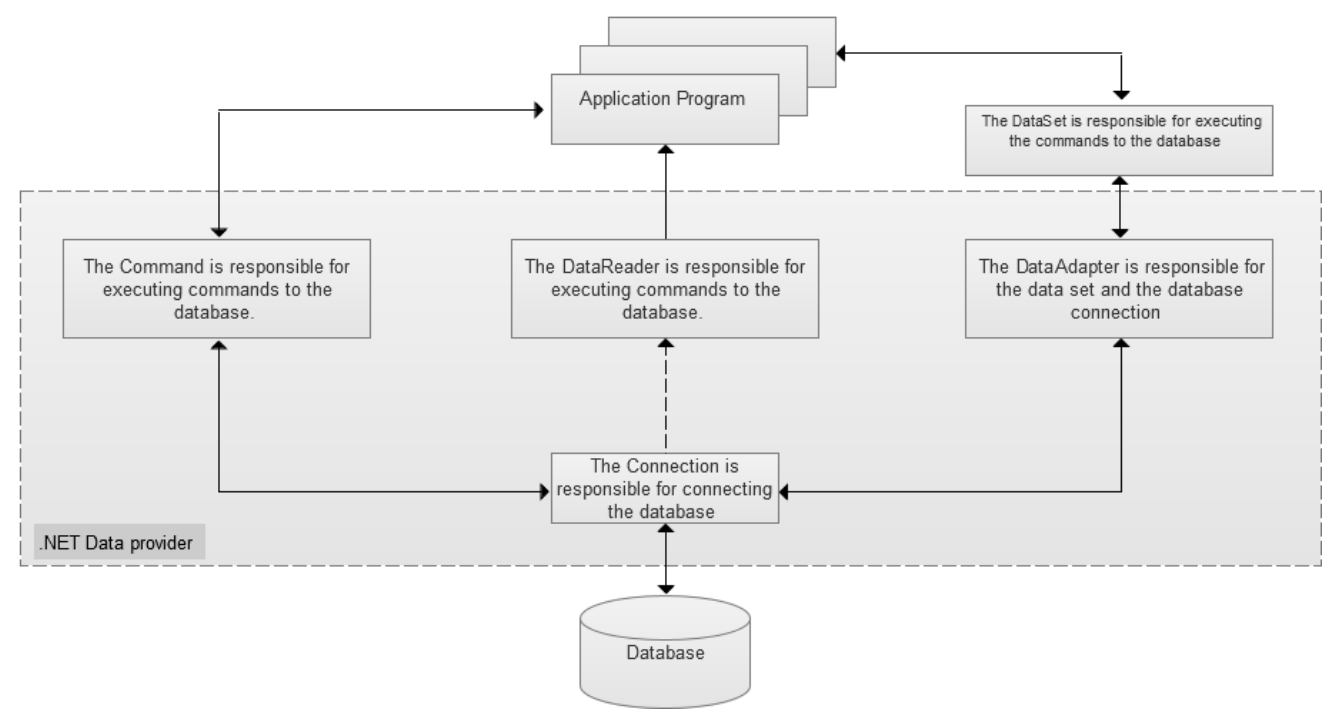

Figure 8.ADO.NET Components

\subsection{Analysis of System Function Module}

Based on the above analysis, we can design a functional block diagram shown in Figure 9. The entire stadium management system is divided into five major functional structures:site management module, management module sparring, member management module, the device management module and system management module. Site management module enables manage inter-ordinary venues and package, sparring management is able to achieve sports consumption in coach training various projects and other services, membership management mainly manage the basic information and consumer information, subscription information of members, enabling customers to arrange sports consumption, equipment management program is required to achieve manage the various venues sports venues, infrastructure, equipment and sports 
equipment effective

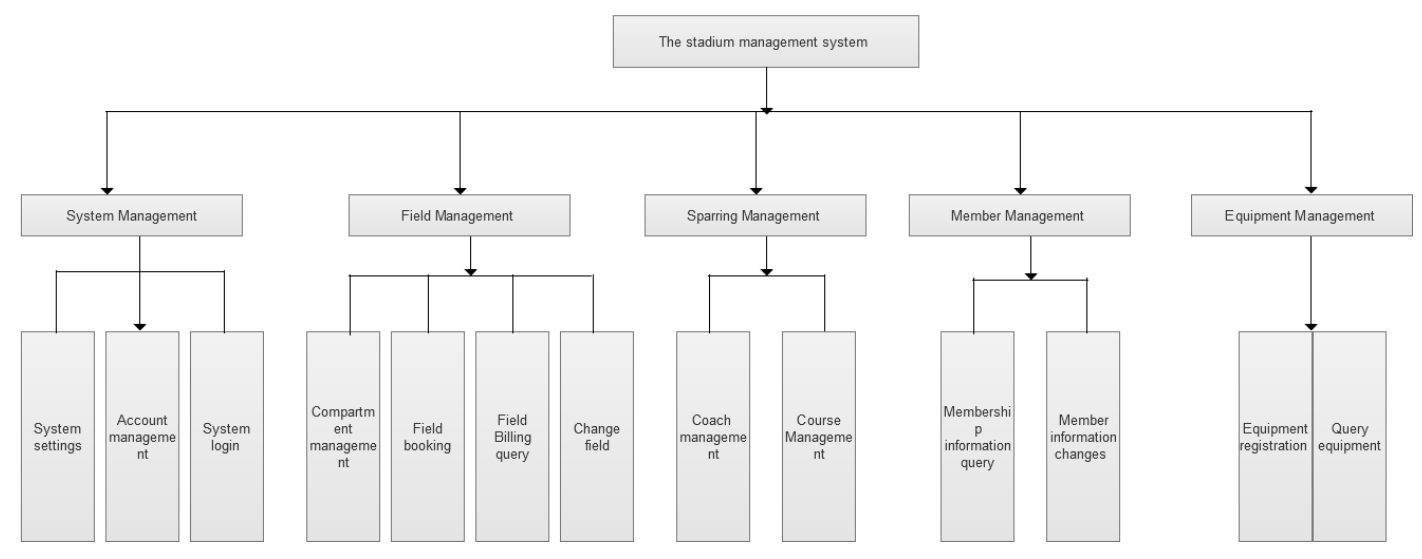

Figure 9. Stadiums Function Block Diagram

System management module: it main achieve to manage entire system which about landing, exit, permissions, roles, and account. Including coaches' maintenance information, press releases and a healthy sense of release.

Sparring Management Module: includes personal information to view, modify, add, and manage personal trainer.

Site Management Module: this module needs to involve the analysis of specific consumption between venues package information.

Member Management Module: includes personal information to view, modify, predetermined personal trainers, and course selection between predetermined packages.

Device Management Module: this is used to register and storage costs for fitness equipment billing.

\subsection{E-R Diagram Database Design}

Preliminary design of the system can be drawn between the E-R entity relationship diagrams ${ }^{[7]}$.the system includes a member, venues, coaching and curriculum analysis of the four entities according to the above requirements. The relationship between them as shown in Figure 10:

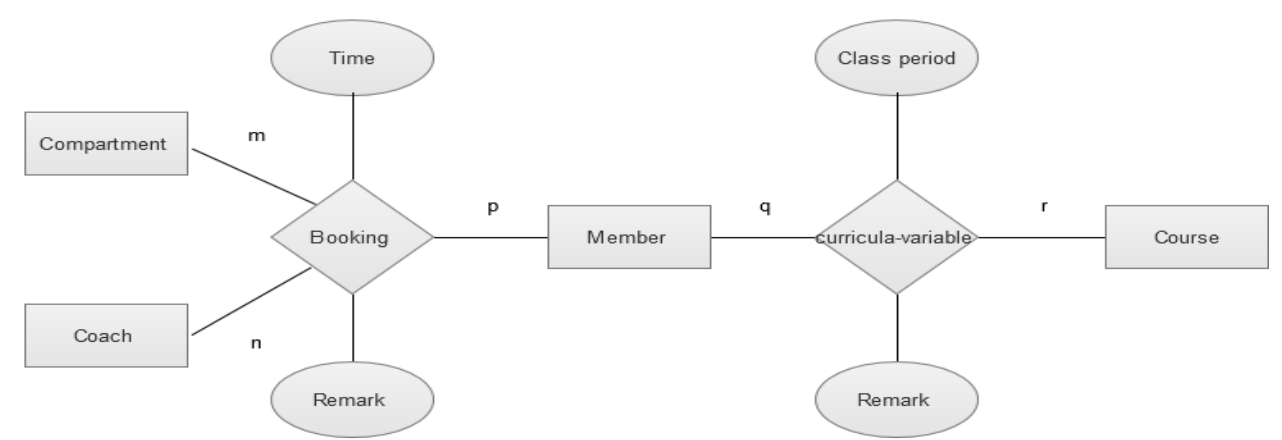

Figure 10. Entity-relationship between Systems

Stadium management information system entity relationship model (E-R diagram) shown in Figure 11: 


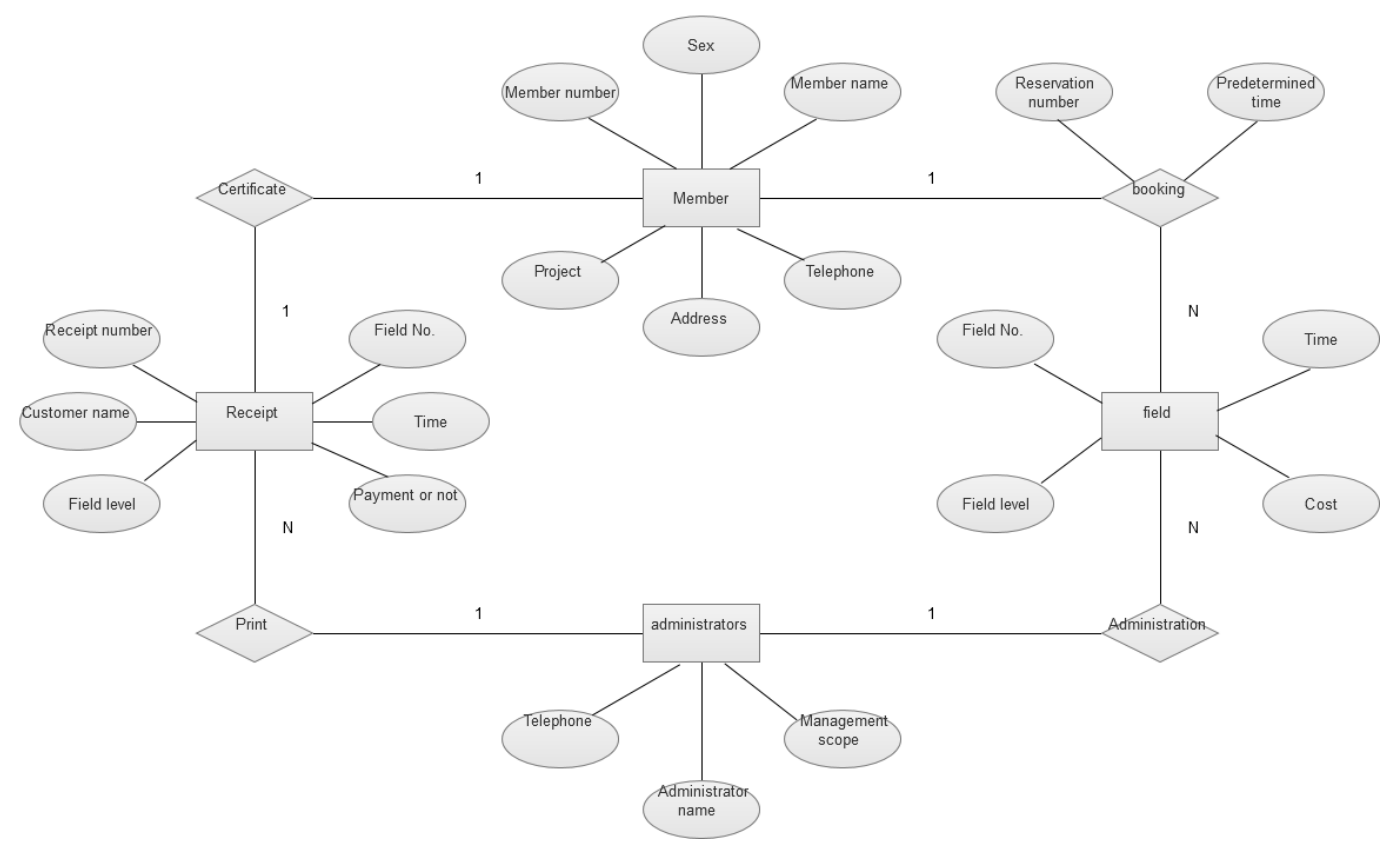

Figure 11. System E-R Diagram

\section{The System Module Function Realization Achieve}

\subsection{System Management Module Functions}

System management module mainly includes user management, log management, system log and exit, the module also includes maintenance and publication of information such as information functions. In this module need to help manage their daily work, and also play a role in making use of ASP.NET language design relevant interface on password maintenance . Log screen program flow chart shown in Figure 12. 


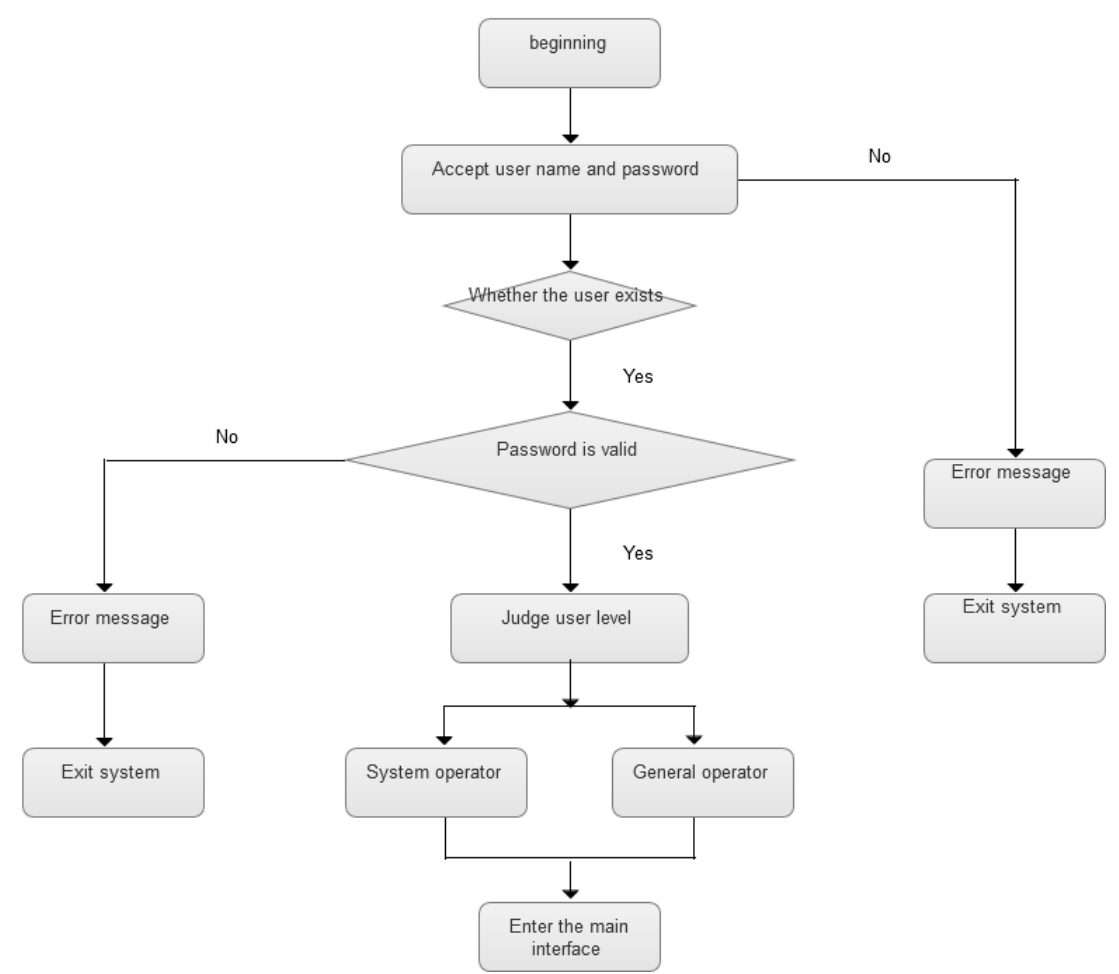

Figure 12. User Log Process Flowchart

If you want to enter the system, it requires not only the correct account also requires a password correctly. If log multiply, passwords, and account number not the right, the client will not be able to continue to log on to the system, the system will prohibited the ranks included the account. Under the user to change the password, it is necessary to enter a password, in order to ensure the accuracy of the password it is also need to confirm it once more, after determining you would have completed the desired action. When we click to find personal information, it is need to query information, and then you can get the required information. At the same time, the system needs to open an account, which must provide their own detailed information, particularly their accounting information. If you need to report the loss of customers and cancel account and other operations also requires the correct password before they can complete.

There are six pages to display user information: user Management.aspx page displays users' information, editUser.aspx page edit users' information; addUser.aspx page adds users' information;ModifyUseInfocompleteusers' information editing ;DeleteUserInfoByIddeletet users' information by users' ID; AddUserInfo complete the operation that add users' information.

At the page we can display mechanism which + tab management control AspNetPager third-party paging controls with GridViewdata.User can control the number of data displayed on the every page, and the default is 5 per page. You can manually enter the number of pages you want to jump.

\subsection{Site Management Module Functionality}

Site management module includes the following aspects: management of private rooms, including room management and repair information change. Site billing interface as shown in figure 13. First of all, we need to choose "for state space, through the menu selection" visitor registration, site billing then entered to billing interface. After entering the interface, you can see the supply and demand of different venues and the cost of 
thesituation.Finally click OK to complete the operation, and click Cancel to cancel the work $^{[8]}$.

The main venue management code module as follows:

baojianxinxi(bianhao,mingcheng,daxiao,leixing,zuidixiaofei,beizhu,zhuangtai)

values('"+bianhao.Text.ToString().Trim()+"','"+mingcheng.Text.ToString().Trim()+"','"+daxiao.Text.To String().Trim()+"','"+leixing.Text.ToString().Trim()+"','"+zuidixiaofei.Text.ToString().Trim()+"','"+bei zhu.Text.ToString().Trim()+"','"+zhuangtai.Text.ToString().Trim()+"') "; int result;

result $=$ new Class1().hsgexucute(sql);

if (result $==1$ )

\{

Response.Write("<script>javascript:alert('Add Success'); </script>");<smiles>C1CCC1</smiles>

else

\{

Response.Write("<script>javascript:alert('System error, check the connection of database);</script $>$ ");

\subsection{Achieve Membership Management Module Functions}

In Member management module,there are the following works included: examination and modification of personal information, as well as coach predetermined site, consumption management. Only need to click on "View $\rightarrow$ consumer consumption status",you can enter the "personal consumption detail query" window.

Member management module main code is as follows: Session["username"].ToString().Trim() + "'";

DataSet result $=$ new $\operatorname{DataSet}()$

result $=$ new Class1 ()$\cdot$ hsggetdata(sql);

if (result != null)

\{

if (result.Tables[0].Rows.Count>0)

baojianbianhao.Text

values('"+baojianbianhao.Text.ToString().Trim()+"','"+jiaolianming.Text.ToString().Trim()+"','"+beiz

hu.Text.ToString().Trim()+"') ";

int result;

result $=$ new Class1().hsgexucute(sql);

if (result $==1$ )

\{

Response.Write("<script>javascript:alert('Add Success'); </script>");

\}

else

Response.Write("<script>javascript:alert('System error, check the connection of database'); $<$ script $>$ ");

\}

\subsection{Sparring Achieve Management Module Functions}

Sparring management module includes a query,modify personal information,and manage private coach.

Sparring management module functioning code is as follows:

if Data.Query2.RecordCount $<1$ then

begin

Application.MessageBox('Service name does not exist, please re-enter.','prompt',64);

Exit;

end;

with Data.Query2 do

begin

SQL.Clear;

Close;

SQL.Add('select $*$ from $t \_$fwxm where fwmc $=: a$ and jldw $=: b^{\prime}$ );

Parameters.ParamByName('a').Value := Trim(fwmc.Text);

Parameters.ParamByName('b').Value $:=$ Trim(jldw.Text);

Open; 
end;

if Data.Query2.RecordCount $>0$ then

begin

Application.MessageBox('The service already exists corresponding units of measurement.',' prompt',64);

end;

\subsection{Achieve Device Management Module Functions}

The main equipment management is complete the registration of sports equipment storage and accounting functions amount better. Once after the storage device you can use custom components TShapeGrid to complete the information entry work. You can also through the components ofTDBGid toinput, as long as customers TShapeGrid component input related field name and the brevity code will display a list of related information. Procedures need to achieve the amount of statistical work, the custom of the Computerysk function, the function can help to achieve the cycle of statistical functions.

There is a relationship between the list of equipment storage register and employee information table (temployee).When clicking"..."button, the list will display the name of the employee in the t_employee. The missing value of time is the current specific date. Users in the table need to increase name of storage device, and the relevant procedures will find the information of device by adding the information to the basic service table ( $t$ $\mathrm{T}$ _fwjcxx).If finding nothing, it will prompt the service does not exist. If exists, it will appear in the TDBGrid to display the relevant information of devices ${ }^{[9]}$.

EquipmentLendList.aspx page display the borrowing information of equipment.Lend.aspx editEquipment page modify the borrowing information of equipment and facilities.AddEquipmentLend.aspx page increase borrowing information of equipment and facilities. ModifyEquipmentLendcomplete the modification operationson borrowing information of facilities and equipment.DeleteEquipmentLendById complete deleting operation on borrowing information of the equipment and facilities according to the ID of equipment and facilities.AddEquipmentLend complete adding operation on borrowing information of equipment and facilities.4.6Database connection ${ }^{[10]}$.

Before using the database, what needs to be done is to establish the appropriate database links, which will be the basis of the application and background data exchange, and ultimately it achieve the ultimate goal by the data source.

The data source can be seen as the data connection, or a link which can also play a role in communication. Asp.net development have access to database through the link, and after that, you can complete the exchange between information and data. In this program, you can complete access to the database. In order to achieve unity of purpose of access to the database, the data can then code in web.config.

Database connection code:

$<$ configuration>

<appsettings>

$<$ add key="cn" value="server=; uid=sa;pwd=sa;Database= netjszx "/>

$</$ appsettings $>$

$</$ configuration $>$

\section{System Test}

We need to test the function of the stadium management system module, including the system management module, site management module, management module sparring, membership management module and device management module.

\subsection{Test Login}

Once you run the program, it will login windowappears, and at the same time, you can 
connect to the database for testing.

Once the user has not entered the correct user name and password, the system will pop up a window. If the user name and password are correct, it can enter the next step. After exiting the system, log back in again, you can start the next test simply by virtue of the identity of the operator of the system. For example, in the window of administrator, after the operation of adding, it is possible to observe that if the generated record meets the criteria or not, and after performing delete, you need to confirm whether the success of the deletion once again.

\subsection{Query Function Test}

At the work in the form of a query, you need to select a single condition as well as multi-criteria query, if you select a query that is the single condition, you will need to check the input field to the correct position, clicking on the button you can get the query results. If you select a query which is more work conditions, you also need to select the query criteria required in the drop-down menu, you can also enter the relevant fields in the edit box, at last, you also need to click the Query button to get the results, but need to final verification to verify the contents.

Table 1. System Function Test Results

\begin{tabular}{|c|c|c|}
\hline Test Module & Test content & result \\
\hline $\begin{array}{c}\text { System } \\
\text { Management } \\
\text { Module }\end{array}$ & $\begin{array}{l}\text { It enables password changes, permissions and role } \\
\text { management, four exit the system operation, and achieve } \\
\text { to maintenance and management of system on basic } \\
\text { information. Including coaches' maintenance information, } \\
\text { press releases and a healthy sense of release. }\end{array}$ & can be realized \\
\hline $\begin{array}{l}\text { Site Management } \\
\text { Module }\end{array}$ & $\begin{array}{l}\text { When pointing of each sub-menu, you can fill in the content } \\
\text { one by one follow the above, which can be performed } \\
\text { between packet of information and sites to view, modify, } \\
\text { add management, and other functions. }\end{array}$ & $\begin{array}{l}\text { You can } \\
\text { manage the site } \\
\text { and packages } \\
\text { between } \\
\text { projects }\end{array}$ \\
\hline $\begin{array}{l}\text { Membership } \\
\text { Management } \\
\text { Module }\end{array}$ & $\begin{array}{l}\text { When pointing of each sub-menu, you can fill in the } \\
\text { content one by one follow the above, including personal } \\
\text { information can be divided into view, modify, predetermined } \\
\text { personal trainers, private rooms and a predetermined course } \\
\text { selection. }\end{array}$ & can be realized \\
\hline $\begin{array}{l}\text { Sparring } \\
\text { Management } \\
\text { Module }\end{array}$ & $\begin{array}{l}\text { When pointing of each sub-menu, you can fill in the content } \\
\text { one by onefollow the above,including personal information } \\
\text { can be implemented in view, modify, add, and manage } \\
\text { personal trainer. }\end{array}$ & $\begin{array}{l}\text { Implementation } \\
\text { can be operate }\end{array}$ \\
\hline $\begin{array}{c}\text { Device } \\
\text { management } \\
\text { module }\end{array}$ & $\begin{array}{l}\text { It realizes the sports equipment storage register, the amount } \\
\text { of accounting and other functions. }\end{array}$ & can be realized \\
\hline
\end{tabular}

\section{Conclusion}

ASP.NET language has been used to design stadiums intelligent management system, including system management, site management, membership management, sparring management and device management five modules, and design of intelligent management system was tested. The results show that the system which designed simplifies the traditional office methods, improve work efficiency, but also can work to a certain extent refinement. In addition, the operation of the system will not only improve work efficiency, but also can greatly improve the accuracy of the information. Finally, it can achieve the 
remote control for the system and save office hours greatly.

\section{References}

[1] L. Ding, S. Lu and H. Yang, "The Integration and Exploitation of Sport Information Resource Based on the Idea of Digital Olympics", Journal of Nanjing Institute of Physical Education (Social Science), vol. 2, (2009), pp. 017.

[2] R. J. Jin and Y. H. Xiao, "Study on the Glare Problems of the Intelligent Lighting Control System in Modern Stadiums", Advanced Materials Research, vol. 753, (2013), pp. 1988-1991.

[3] Q. H. Li and Y. L. Yu, "The Design and Implementation of the Stadium Safety Evacuation System", Advanced Materials Research, vol. 998, (2014), pp. 1657-1660.

[4] X .S. Zhao and S. Bai, "The Research and Design of the Adaptive Automotive Headlamps Based LED System”, Applied Mechanics and Materials, vol. 742, (2015), pp. 753-757.

[5] Q. H. Li and Y. L. Yu, "The Design and Implementation of the Stadium Safety Evacuation System", Advanced Materials Research, vol. 998, (2014), pp. 1657-1660.

[6] Q. Liu, C. Hu and L. Shen, "Research on Management of Chinese Stadiums", 2014 2nd International Conference on Education Technology and Information System (ICETIS 2014). Atlantis Press, (2014).

[7] S. Lucas, A. S. Afonso and V. Ferreira, "Improving by sustainability in sport facilities", Energy for Sustainability 2013-Sustainable Cities: Designing for People and the Planet, (2013).

[8] L. U. O. Dai, "On modern management mode of college stadiums", Journal of Henan University of Urban Construction, vol. 2, (2013), pp. 021.

[9] G. U. P. J. Wei, "Specialty trend of sports information technology in PE institutes", Journal of Wuhan Institute of Physical Education, vol. 9, (2005), pp. 031.

[10] G. U. O. J. Wei, "Specialty trend of sports information technology in PE institutes", Journal of Wuhan Institute of Physical Education, vol. 9, (2005), pp. 031.

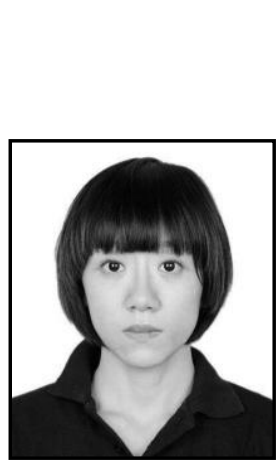

\section{Author}

Jing NING. She was born in Marchof 1981. Now she is a lecturer in Physical Research Department, Hebei University. Her research direction is SportEducation; she has got the degree of master. 
International Journal of Database Theory and Application Vol.9, No.12 (2016) 